Cahiers d'études italiennes

\title{
La théâtralité du Décaméron
}

\section{Claude Perrus}

\section{OpenEdition \\ Journals}

Édition électronique

URL : http://journals.openedition.org/cei/862

DOI : 10.4000/cei.862

ISSN : 2260-779X

Éditeur

UGA Éditions/Université Grenoble Alpes

\section{Édition imprimée}

Date de publication : 15 juillet 2008

Pagination : 5-12

ISBN : 978-2-84310-122-9

ISSN : $1770-9571$

Référence électronique

Claude Perrus, «La théâtralité du Décaméron », Cahiers d'études italiennes [En ligne], 8 | 2008, mis en ligne le 15 janvier 2010, consulté le 20 avril 2019. URL : http://journals.openedition.org/cei/862 ; DOI : $10.4000 /$ cei.862 


\section{LA THÉÂTRALITÉ DU DÉCAMÉRON}

\section{Claude Perrus}

Université de la Sorbonne Nouvelle-Paris 3

La question à la quelle fait allusion le titre de mon intervention couvre deux aires de recherche. D'une part: l'influence du "théâtre» sur le Décaméron, "théâtre" étant entendu dans un sens très large, qui englobe les grands comiques latins (non encore ressuscités sur scène) ${ }^{1}$, les textes plus récités, probablement, que joués, de la comédie élégiaque médiévale, et aussi l'expérience de la lauda drammatica dans le cadre des confréries, les performances des jongleurs et des bouffons, et les pratiques spectaculaires publiques, avec diableries et autres déguisements ${ }^{2}$. D'autre part: l'influence du Décaméron sur le théâtre renaissant (comédie régulière mais aussi tragédie), influence dont les modalités (analysées notamment par Nino Borsellino ${ }^{3}$ ) sont très diverses, et qui se combine souvent avec l'imitation directe des comiques latins ou des tragédies de Sénèque, comme le montre la superposition de Plaute et de Boccace dans la Calandria de Bibbiena ou celle de Sénèque et de Boccace dans la tragédie Orbecche de Giraldi Cinzio.

Cependant, entre ces deux aires, qui relèvent surtout d'une problématique des sources, un autre champ de recherche se déploie: celui du travail de l'écriture. On sait que le Décaméron traverse un grand nombre de genres narratifs pour fonder la nouvelle moderne, ouverte à son tour sur

1. Il faudra attendre certaines initiatives universitaires (comme à Padoue, vers la fin du $\mathrm{XV}^{\mathrm{e}}$ siècle) et l'entreprise de traduction et de représentation scénique de Plaute et Térence par le comédien Francesco de' Nobili, dit Cherea, au XVI' siècle.

2. Par exemple la "diablerie» organisée à Florence sur l'Arno en mai 1304, et qui se termina tragiquement, selon le récit de Giovanni Villani, Nuova Cronica, VIII, 70. Un écho de ce genre de spectacles se trouve dans Dec., IV, 2 (Frère Alberto est exhibé sous un déguisement d' " homme sauvage») et VIII, 9 (Buffalmacco se travestit en diable pour jouer un mauvais tour au médecin Simone da Villa).

3. Nino BORSELLINO, "Decameron come teatro", in IDEM, Rozzi e Intronati. Esperienze e forme di teatro dal "Decameron» al "Candelaio», Rome, Bulzoni, 1976, p. 11-49. 
des formes dont les siècles suivants redécouvriront les potentialités. Citons, entre bien d'autres exemples, le bozzetto vériste avec l'histoire du curé de Varlungo (VIII, 2), le récit policier avec l'histoire de Tedaldo degli Elisei (III, 7).

C'est dans ce même mouvement d'exploration que la «nouvellisation " peut déboucher sur le mime et sur la comédie (comme l'ont montré les analyses de Mario Baratto ${ }^{4}$ ), voire sur la tragédie. Cette dramaturgie-là ne s'autorise pas toujours de sources ou de cautions théâtrales, elle peut naître aussi dans le champ même de l'expérimentation narrative, par la théâtralisation de modèles non théâtraux ou d'une expérience empirique qui relève de la chronique quotidienne.

Il convient toutefois de souligner que ce travail sur des formes passées ou cette expérimentation de formes futures ne visent pas un «au-delà » de la nouvelle. L'intention, la tension de Boccace visent avant tout à inscrire ces formes virtuelles dans le récit, c'est-à-dire à inscrire une possible dramaturgie dans le cadre rigoureux de la narration courte.

C'est pourquoi, pour passer du récit théâtralisant (celui de Boccace) au récit théâtralisable, objet d'une réutilisation par les dramaturges, il faut que ces derniers procèdent à une déconstruction, toujours périlleuse et souvent réductrice, de l'organisme "nouvelle» institué par Boccace. Je traiterai parallèlement de ces deux aspects.

Rappelons tout d'abord quelques-uns des modes ou facteurs élémentaires de théâtralisation (quelle que soit la nature du texte source), qui autorisent une "transcodification " ultérieure du récit, réalisée ou non par des dramaturges mais s'opérant de toute façon, au cours de la lecture, dans la tête du lecteur.

En premier lieu, par rapport aux narrations précédentes comme par rapport à des formes plus modernes, plus éclatées, du récit court, le schéma (j'allais dire le scénario) de la nouvelle de Boccace. Sauf exception (par exemple dans le cas de certaines nouvelles en deux parties, comme celle de Cimone, $\mathrm{V}, 1$ ) Boccace observe une disposition rigoureuse, à savoir une exposition, un nœud de l'action (avec diverses péripéties) et un dénouement, parfois assorti d'un épilogue (qui clôt l'histoire en résumant le futur des personnages), ou même d'un commentaire du conteur qui, réintégrant le conte dans la fictive oralité de la brigata, devient en quelque sorte l'équivalent du «plaudite et valete» théâtral. Cette disposition, dont l'économie n'est pas entièrement imputable à l'ars dictandi, correspond par 
avance à celle d'une «intrigue" régulière, et il est significatif que lorsque $M^{\text {gr }}$ Bonciani, en 1574, expose à une académie florentine les principes de la nouvelle idéale - c'est-à-dire boccacienne -, il se fonde explicitement sur la définition de la tragédie par Aristote pour dégager, à partir des cinq parties de la tragédie, les trois parties de cette nouvelle (prologo, scompiglio, sviluppo).

Il en va de même de la distribution rationnelle des informations, au début et en cours de récit, informations qui seront fonctionnalisées de manière qu'à la fin il ne reste, du moins en principe, aucun résidu non exploité. Cette économie narrative coöncidera elle aussi avec les exigences des pièces régulières du XVI siècle, pièces d'ailleurs souvent précédées d'un «argument» récité qui souligne la parenté originelle entre les deux types de fiction.

À l'intérieur de cette disposition, donc, qui réduit et recompose drastiquement les données fournies par les sources éventuelles, il va de soi que certaines trames se prêtent, plus que d'autres, à une transcodification dramaturgique. Cela tient avant tout aux deux paramètres de l'espace et du temps. Les navigations, chevauchées, enlèvements et autres aventures supposant une multiplicité de lieux et une longue durée sont difficilement transposables, du moins sur la scène statique, sans procédés illusionnistes et changements de décor, qui domine en Italie jusque vers la fin du $\mathrm{XVI}^{\mathrm{e}}$ siècle. Ils le deviendront dans la scénographie ultérieure (notamment à l'opéra), de même que dans le théâtre élisabéthain: le Cymbeline de Shakespeare, par exemple, reprend (mais en réduisant les déplacements et le nombre de lieux) l'aventure de Gilette de Narbonne (III, 9). On objectera que certains épisodes, à défaut d'être montrés, peuvent être racontés sur scène, mais le procédé a des limites évidentes. Et surtout ce type d'action aventureuse fait l'objet, dans le Décaméron, d'une expérimentation romanesque, voire épique, qui en exploite peu les potentialités dramatiques.

Il en va différemment des nouvelles où un temps plus limité et un nombre plus réduit de protagonistes et de lieux mettent en évidence une "mécanique» visualisable. Ainsi le château de Tancrède et surtout la chambre de Ghismonda, avec ses trois protagonistes, suggèrent un modèle de huis clos tragique, que réalisera (comme je l'ai dit) Giraldi Cinzio en 1541 dans Orbecche, transposition très sénéquienne et sanglante de la nouvelle IV, 1 . Je pourrais citer d'autres châteaux tragiques, comme celui de Guillaume de Roussillon, mais je me contenterai de renvoyer, sur ce point, aux travaux de Vittorio Russo. 
Mais il existe aussi le huis clos comique, dont un cas limite se rencontre dans la nouvelle IX, 6 (dite "du berceau»). La chambre de l'hôte où dorment (ou veillent) cinq personnes - sans compter le chat - est le lieu d'une trame circulaire de permutations et de quiproquos nocturnes: c'est le cadre virtuel (non exploité, à ma connaissance) d'une comédie de Feydeau.

Cependant l'espace le plus commun des trames comiques est celui de la rue (le dehors) avec des maisons (le dedans), lieu de rencontre des personnages, de leurs apparitions et disparitions. Sur le versant narratif, cette rue, c'est la ville (à la fois décor, occasion, et moteur de trames très variées). Sur le versant théâtral, ville et rue se configureront comme un espace plus neutre et fonctionnel, celui des comédies de Plaute et de Térence, qu'utilisera la scène renaissante, du moins à ses débuts.

Après ces quelques remarques préliminaires il est temps de relever quelques composants du matériau théâtralisant/théâtralisable. Je m'en tiendrai à la comédie, car c'est dans la comédie, comme on le sait, que les virtualités théâtrales du recueil ont été le plus exploitées. Pour expliquer ce phénomène, il n'est peut-être pas inutile de revenir un instant sur la nature du comique boccacien.

Le Décaméron opère, entre autres révolutions, une libération du comique, cantonné jusqu'alors dans des genres et sous-genres inférieurs, qu'ils relèvent ou non du spectacle: farce, sotie, dits des jongleurs, fabliaux, textes parodiques tels que les sermons joyeux, par exemple, etc.

Les éléments comiques de tous ces textes font l'objet, de la part de Boccace, d'une récupération que l'on peut qualifier de généreuse. Prenons les récits de beffe: il est très significatif que ces nouvelles relèvent d'un programme proposé, et même imposé, par Dioneo à la fin de la sixième journée. Cette initiative de Dioneo sanctionne l'intégration d'un certain type de rire «bas» à l'expérience de vie de la brigata courtoise. En même temps, elle récupère et "rachète» l'altercation des serviteurs (qui a lieu comme un coup de théâtre au début de cette même journée VI), altercation qui, vue et entendue au premier degré, était propre à susciter chez les témoins une curiosité malsaine, une bassa voglia. Ecourtée par le même Dioneo, elle ne lui suggère pas moins, à distance, une materia da ragionare pour la journée de sa royauté.

Mais le comique entre aussi, selon des proportions variables, dans la composition d'autres récits, où il peut acquérir des nuances inédites allant jusqu'à l'humour ou au nonsense modernes. En d'autres termes le comique et son effet: le rire, appartiennent désormais de plein droit à la nature même des choses, dans toute l'amplitude de leur "narrabilité5». 
Venons-en donc à quelques traits de cette comédie virtuelle. La variété des types et des situations du livre est d'abord fonction de la variété des textes dont il s'inspire. Le miracle, c'est que leur mise en nouvelle (avec sa charge éventuellement théâtrale) arrive à les faire coexister à l'intérieur d'une vaste gamme de récits, sur la base d'un pacte de vraisemblance conclu entre l'auteur et son lectorat potentiel.

Un premier répertoire de personnages-types provient de la comédie latine, où il est le plus souvent lié à un scénario d'amours contrariées puis réalisées. Aux héros (entendons: les amoureux) s'opposent divers types de vieillards sourcilleux; les auxiliaires des héros sont des serviteurs ou servantes ou confidents qui manipulent les ennemis; d'autres comparses peuvent intervenir (le pédant, le sot, le parasite, le bouffon...). Amoureux mis à part, ces acteurs étaient censés correspondre, à l'origine, à des caractères intemporels (sur le modèle mis en scène par Théophraste dans ses micro-récits) et par suite à des «emplois».

Sans toucher aux emplois, Boccace varie et actualise les "caractères". C'est ainsi que le senex iratus, dont le prototype tragique est Tancredi (IV, 1 ), peut se réincarner entre autres dans le sourcilleux mais débonnaire Lizio da Valbona (nouvelle V, 4 dite «du rossignol»). Le médecin Simone da Villa, pour sa part, cumule les traits du pédant et de l'imbécile, voire du bouffon malgré lui (quand il fait montre de ses talents de chanteur), mais il change de fonction lorsqu'il devient le complice d'une farce jouée à Calandrino (IX, 3). De même les serviteurs présentent un échantillonnage surprenant de macchiette et même de personnalités, de l'habile et éloquente Lusca (VII, 9) à l'inénarrable Guccio $\operatorname{Imbratta}^{6}$ (VI, 10), que je n'ai pas le temps d'énumérer.

Ces innovations doivent beaucoup, comme l'on sait, à la comédie élégiaque $^{7}$ et aux fabliaux, qui ont enrichi le répertoire des types, en relation avec des situations nouvelles, dont la plus répandue est l'adultère, peu présent dans la comédie classique mais déjà abondamment illustré par des narrateurs tels qu'Apulée, dont Boccace s'inspire directement. L'incre-

5. C'est pourquoi je récuse l'application par M. Bakhtine, au Décaméron, de la catégorie du " carnavalesque " conçu comme alternative à la culture officielle et à ses réseaux de pouvoir. La subversion de Boccace est plus subtile. Voir M. Mikhaïl BaKhtine, L'œuvre de François Rabelais, Paris, Gallimard, 1970, p. 271-272.

6. Guccio Imbratta est issu d'un valet de la comédie élégiaque nommé Spurio (Nomina sunt...).

7. Michelangelo PiCONE, entre autres, a fourni une riche analyse comparative de la Comedia Lydiae d'Arnoul d'Orléans, voir "La Comedia Lidiae dallo Zibaldone al Decameron" in Gli Zibaldoni di Boccaccio. Memoria, scrittura, riscrittura, éd. M. Picone \& C. Cazalé-Bérard, Florence, Franco Cesati, 1998, p. 401-414. 
vable triangle érotique (le mari, la femme et l'amant) se prête à de nombreuses variations et convoque non seulement des figures inédites d'amoureux (des ecclésiastiques par exemple) mais aussi des rôlesfonctions promis à un bel avenir théâtral. Je ne citerai que l'entremetteuse, en provenance des Métamorphoses d'Apulée, dont Boccace fait un véritable personnage avec sa "santa Verdiana" de la nouvelle V, 10. La Lena de l'Arioste sera son héritière directe, et la Syra de Térence paraît bien pâle à côté de ses deux consœurs. Je reviendrai dans un instant sur son discours.

Le temps me manque pour évoquer de façon précise les divers "scénarios» de comédie que présente le Décaméron. Je voudrais cependant évoquer ce vecteur du théâtre qu'est la parole vive (monologues et dialogues des personnages). Dans un texte narratif, cette parole relève en principe de la mimésis, mais pas nécessairement d'une mimésis dramaturgique, car elle est liée à d'autres paramètres, commentatifs, diégétiques ou descriptifs, alors qu'au théâtre elle est, outre que moyen de caractérisation, avant tout action. Elle joue évidemment un grand rôle dans le récit théâtralisant de Boccace, et c'est là, d'ailleurs, que le traitement des sources est le plus immédiatement visible, soit que Boccace introduise de la parole là où il n'y en avait pas, soit qu'il aménage, avec des modifications surprenantes, la parole existante.

Passons rapidement sur la parole-action, où la stratégie verbale de certains personnages détermine le cours de l'intrigue: de Ciappelletto (I, 1) à tous les autres manipulateurs des nouvelles, pour nous attarder en revanche sur un phénomène bien connu, l'extension des registres linguistiques, depuis l'éloquence ornée jusqu'aux niveaux dialectaux et idiolectaux les plus variés: un véritable dictionnaire est proposé par Boccace aux futurs comédiographes. Le choix de tel ou tel langage relève, bien entendu, du principe de la convenientia, et sert en même temps à la typisation (commune au récit et au théâtre). Mais de surcroît Boccace, à travers cette typisation, réussit à faire réellement entendre des voix. Je m'en tiendrai à deux exemples.

Le discours de la "santa Verdiana» $(\mathrm{V}, 10)$ réoriente et reformule entièrement l'interminable bavardage de l'entremetteuse d'Apulée - et sans doute les propos assertifs de la Vieille, dans le Roman de la Rose, sont pour quelque chose dans cette condensation. Il en ressort un monologue scénique autonome, farci de proverbes et nourri d'une réjouissante mauvaise foi, auquel il n'y a rien à ajouter ou à retrancher.

La célèbre tirade de la belle-mère d'Arriguccio (VII, 8) constitue une véritable scène: devant le public familial réuni, elle laisse éclater son indi- 
gnation contre son gendre médusé et s'enivre de sa propre parole. On ne peut, à la lecture, dissocier cette tirade d'un accent florentin que rien dans la graphie n'indique mais qui est induit par le rythme même du texte. Boccace fait vivre là aussi un type nouveau, celui de la mégère (non apprivoisée), une mégère socialement définie, et dont le discours dément comiquement les prétentions nobiliaires.

De même certains dialogues, fonctionnels à une simple animation ou variation du récit, deviennent potentiellement dramatiques dès lors qu'ils impliquent (comme l'a montré Pamela Stewart ${ }^{8}$ ) la technique du double destinataire: l'interlocuteur et un témoin (comme dans la fausse confession de Ciappelletto (I, 1), destinée aussi à ses deux compatriotes cachés), ou encore l'interlocuteur et le lecteur, qui fait alors fonction de public (voir certains dialogues surréalistes tels que celui de Ferondo avec le moine qui le garde au "purgatoire», III, 8).

Enfin, dans d'autres cas, l'émancipation du langage par rapport à son objet même, comme à toute fonction de communication, peut faire de la parole un pur spectacle. L'improvisation de Frère Cipolla (VI, 10), d'ailleurs à double destinataire elle aussi, excède les limites pourtant vastes $\mathrm{du}$ "sermon joyeux». De même les inventions verbales, les tautologies absurdes de Bruno et Buffalmacco annoncent, dans certaines pièces de l'Arétin, le déploiement d'une véritable pathologie du langage: dans la Cortigiana messire Maco, un mélange de maître Simone da Villa et de Calandrino, tient des propos absolument insensés.

Tels sont quelques-uns des aspects qui manifestent cette «libération du comique» qui contribuera largement à rendre possible la restauration de la comédie à la Renaissance. Le Décaméron irrigue tout ce théâtre, à commencer par son vocabulaire, qui y fonctionne aussi comme citation, dans une complicité manifeste avec le public.

La dite comédie, cependant, en dehors de ces pratiques citationnelles, n'utilisera guère le Décaméron que par l'évocation allusive de personnages, et des prélèvements d'intrigues et de situations mais le plus souvent (me semble-t-il) de scènes isolées souvent réservées à des sous-intrigues parallèles à l'action principale. Inévitablement, de telles opérations «déproblématisent» et «démoralisent» le texte source, mais c'est la rançon de toute recodification.

8. Pamela D. STEWART, «Il testo teatrale e la questione del doppio destinatario", in Retorica e mimica nel "Decameron" e nella commedia del Cinquecento, Florence, Olschki, 1986, p. 103-123. Voir aussi Tatiana CrIVELli, Teresa NoCITA, "Teatralità del dettato, stratificazioni culturali, plurivocità degli esiti: il Decameron fra testo, ipertesto e generi letterari", in Autori e lettori di Boccaccio. Atti del Convegno internazionakle di Certaldo (20-22 sett. 2001), Florence, Franco Cesati, 2002, p. 209-233. 
Il faut cependant rappeler un car limite, celui de l'Arétin qui, dans le Filosofo, introduit sous forme d'intrigue parallèle la nouvelle d'Andreuccio (II, 5) en version intégrale. Certains épisodes, difficilement représentables, sont résumés en monologue par le héros lui-même, lequel ne s'appelle plus Andreuccio mais... Boccaccio, devenu pérugin: une métamorphose que le vrai Boccace aurait modérément appréciée.

Je n'ai pas évoqué - tout le monde l'aura remarqué - la structure portante des nouvelles, et plus précisément cette partie de la structure constituée par la brigata. À mes yeux en effet, plus qu'une mise en scène des nouvelles, cette chorégraphie des journées, à la scansion très régulière, quasi rituelle, clôturée par des chants et des danses, est une mise en scène des instances de la narration, qui impose le point de vue d'un «intellectuel collectif", en même temps qu'elle fixe un mode de fruizione des récits.

Mais les dix acteurs de ce spectacle peuvent être récupérés, au prix de transformations radicales, dès lors qu'on se propose de représenter plusieurs histoires du Décaméron, qu'il faut alors... réencadrer! Cela a été le cas notamment en 1971 et en 2001, à l'initiative des metteurs en scène Jacques Lassalle puis Jean Boilot. Dans cette opération, les devisants (aussi pour des raisons économiques de distribution!) sont tour à tour narrateurs et acteurs de leurs propres récits. Par une fructueuse trahison, la brigata peut et doit alors se transformer en un groupe rassemblant diverses classes sociales, créant ainsi un jeu de miroirs inédit entre narrateurs et nouvelles.

De telles opérations prouvent bien que le Décaméron, comme machine virtuelle à produire du spectacle, ne cessera jamais de nous étonner.

Je concluerai ce propos comme au théâtre : Valete. 